\title{
Bond strength to root dentin and fluid filtration test of AH Plus/gutta-percha, EndoREZ and RealSeal systems
}

\author{
Alaa Abdul MAHDI', Victoria BOLAÑOS-CARMONA², Santiago GONZALEZ-LOPEZ ${ }^{3}$
}

1- PhD student, Department of Stomatology, School of Dentistry, University of Granada, Granada, Spain.

2- PhD, MD, Associate Professor, Department of Pediatric Dentistry, School of Dentistry, University of Granada, Granada, Spain.

3- PhD, MD, Associate Professor, Department of Operative Dentistry, School of Dentistry, University of Granada, Granada, Spain.

Corresponding address: Santiago Gonzalez-Lopez - Faculty of Dentistry, Department of Operative Dentistry - Colegio Máximo s/n. Campus Universitario de Cartuja, University of Granada - E-18071 - Granada - Spain - Phone: 958243803 - Fax: + 34958240908 - e-mail: sglopez@ugr.es

Submitted: January 11, 2013 - Modification: May 3, 2013 - Accepted: June 20, 2013

\section{ABSTRACT}

\begin{abstract}
bjectives: To investigate the bond strength and seal ability produced by AH Plus/guttapercha, EndoREZ and RealSeal systems to root canal dentin. Material and Methods: Sixty extracted single-root human teeth, instrumented manually to size 40, were divided into three groups $(n=20)$ according to the sealer used; G1: AH Plus, G2: EndoREZ, and G3: RealSeal sealers. After filling using the lateral condensation technique, each sealer group was randomly divided into two subgroups according to the tests applied ( $n=10$ for $\mu$ Push-out test and $n=10$ for fluid filtration test). A fluid filtration method was used for quantitative evaluation of apical leakage. Four 1-mm-thick slices (cervical and medium level) were obtained from each root sample and a $\mu$ Push-out test was performed. Failure modes were examined under microscopy at 40x, and a one-way ANOVA was applied to analyze the permeability. Non-parametrical statistics for related (Friedman's and Wilcoxon's rank tests) or unrelated samples (Kruskal-Wallis' and Mann-Whitney's tests) allowed for comparisons of $\mu$ Push-out strength values among materials at the different levels. Statistical significance was accepted for $p$ values <.05. Results: There are no significant differences among fluid filtration of the three sealers. The sealer/core material does not significantly influence the $\mu$ Push-out bond strength values $(F=2.49 ; p=0.10)$, although statistically significant differences were detected with regard to root level $\left(\mathrm{Chi}^{2}=23.93 ; \mathrm{p}<0.001\right) . \mathrm{AH}$ Plus and RealSeal obtained higher bond strength to intraradicular dentin in the medium root slices. Conclusions: There are no significant differences between the permeability and global $\mu$ Push-out bond strength to root canal dentin achieved by AH Plus/gutta-percha, EndoREZ and RealSeal systems.
\end{abstract}

Key words: Resin cements. Root canal filling material. Compressive strength. Leakage.

\section{INTRODUCTION}

The fundamental goals of endodontic therapy are to achieve successful cleaning and shaping of root canals and a hermetic apical seal ${ }^{25}$. An ideal root canal sealer should adhere to both dentin and the core filling material. Methacrylate resin-based sealers, based on dentin adhesion technologies, have been developed in an attempt to seal the root canal system more effectively, improving bonding to radicular dentin ${ }^{26}$, but their utilization requires removal of the smear layer and collagen exposure, because retention is largely achieved by micromechanical interlocking between collagen matrix and resin ${ }^{29}$. Ethylenediaminetetraacetic acid (EDTA) has been widely used for this purpose as the final irrigant before applying methacrylate resin-based sealers ${ }^{5,13}$; however, the results were poor regarding its bond strength adhesion to root dentin, whereas the non-bonding $\mathrm{AH}$ Plus sealer presented greater adhesion $7,10,17,18$.

No correlation has been found between apical microleakage and sealer bond strengths to intraradicular dentin in vitro ${ }^{1,2}$. While we assume 
that preventing effective microleakage is perhaps more important for endodontic application than bond strength ${ }^{26}$, there is no universally accepted method for the evaluation of leakage ${ }^{33}$. Each has its limitations, being governed by electrical, filtration and/or diffusion laws ${ }^{24}$. Comparisons between different methods point to contradictory results, and, therefore, questionable clinical relevance ${ }^{12,24}$.

Push-out bond strength testing has become a common method for determining the effectiveness of adhesion between endodontic materials and intraradicular dentin. Zicari, et al. ${ }^{34}$ (2008) have correlated the push-out bond strength and sealing ability with the fluid filtration method of adhesive cements routinely used for fiber-post bonding, but to date there is no documented correlation using methacrylate resin-based sealers. We believe that this aspect is very important, because any filtration can hydrolytically degrade the adhesive interface. Hence, the purpose of this in vitro study was to quantitatively assess the bond strength to dentin root canal and the sealing properties of two methacrylate resin-based sealers and their corresponding core points (RealSeal and EndoREZ), then compare them with the gold standard conventional nonbonding $\mathrm{AH}$ Plus/gutta-percha. The $\mu$ Push-out test and fluid filtration method were used to test the null hypothesis that there is no difference in the bond strength and sealing properties of RealSeal, EndoREZ systems and $\mathrm{AH}$ Plus/gutta-percha.

\section{MATERIAL AND METHODS}

We used 60 human anterior teeth, recently extracted for periodontal reasons from anonymous subjects under a protocol ( $n^{\circ} 11 / 2011$ ) approved by the Ethics Committee of the School of Dentistry, University of Granada. All teeth had single straight root canals and closed apices and none showed caries lesions or had received restorative or root canal treatment. After extraction, any calculus and soft tissue was removed and they were stored in $0.1 \%$ thymol diluted in distilled water at $4^{\circ} \mathrm{C}$ for use in this study within 3 months following extraction.

The crowns of the teeth were cut perpendicular to the long axis of the root at the cement-enamel junction with a diamond coated disk at slow speed with constant water-cooling. Root canals were instrumented using the step back technique to obtain a flared preparation. In all teeth, the working length was determined visually by subtracting $1 \mathrm{~mm}$ from the length of a \#10 K-file (Dentsply Maillefer, Ballaigues, Switzerland) at the apical foramen. Biomechanical preparation was performed manually with K-files until size \#40. After each step in the flare preparation, the canal was irrigated with $2.5 \%$ sodium hypochlorite during $1 \mathrm{~min}$. Then, 17\% EDTA was used as a final irrigant for 1 min, after a rinse of distilled water ( $1 \mathrm{~min}$ ) to remove any remnants of the irrigating solutions. The canals were dried using paper points.

The roots were randomly divided into three equal groups $(n=20)$ according to the root canal sealer used; G1: AH Plus (Dentsply Maillefer, Ballaigues, Switzerland), G2: EndoREZ (Ultradent Products, Inc., South Jordan, Utah, USA) and G3: RealSeal (Sybron Endo, Glendora, CA, USA).

For the group filled with AH Plus, the sealer was mixed using $\mathrm{AH}$ Plus jet mixing system, and then introduced into the root canal orifices with the intraoral tip. A 40.02 taper master gutta-percha cone was placed into the canal and then the canal was filled up using the cold lateral condensation technique and accessory gutta-percha points size \#25 and \#20 dipped in a small amount of sealer.

EndoREZ was mixed in an ultra-mixer and dispensed using a narrow diameter syringe (Skini Syringe) (Ultradent Products Inc., South Jordan, Utah, USA) with a fine-tipped cannula Navi Tip (Ultradent Products, Inc., South Jordan, Utah, USA). Roots were filled totally with EndoREZ sealer. Then a 40.02 resin coated gutta-percha cone (EndoREZ point) (Ultradent Products, Inc., South Jordan, Utah, USA) was placed inside the canal, and additional EndoREZ points size \#25 and \#20 were placed into the canal using the cold lateral condensation technique.

For the group filled with RealSeal, RealSeal Primer (SybronEndo, Glendora, CA, USA) was introduced into the root canal with a paper point (Roeko, Langenau, Germany) soaked with the primer for the apical region. The primer was placed into the middle and coronal part using a micro brush (Microbrush International, Grafton, WI, USA). After 30 seconds, excess primer was removed with paper points. The sealer was dispensed directly from the tip of its auto mix dual-chamber syringe, according to the manufacturer's instructions. A 40.02 taper RealSeal master point was placed into the canal and then additional RealSeal accessory points size \#25 and \#20 were placed into the canal using the cold lateral condensation technique.

In all specimens, the excess of points was removed with a hot instrument and the remainder was condensed vertically. They were resected at the apex perpendicular to the long axis of the tooth until $10 \mathrm{~mm}$ of root length was obtained. Each root was transferred to a test tube (Eppendorf Ibérica, Madrid, Spain) and stored in a humidifier at $37^{\circ} \mathrm{C}$ for 24 hours to complete sealer setting. All preparation and obturation procedures were done by one operator.

Finally, each sealer group $(n=20)$ was randomly divided into two subgroups according to tests applied ( $n=10$ for $\mu$ Push-out test and $n=10$ for fluid 
filtration test).

\section{Leakage evaluation}

The external root surfaces of subgroups for the fluid filtration test were covered with a layer of nail varnish leaving the coronal and apical orifices open. The specimens were attached to a device to measure the permeability by fluid transport described by Pashley, et al.22 (1988). The roots were inserted into a silicon tube $(2 \mathrm{~mm}$ internal diameter), and attached with cyanoacrylate glue on the outer surface of the tube. A pressure tank of $250 \mathrm{ml}$ of distilled water was placed $100 \mathrm{~cm}$ higher than the root to create a pressure of 100 $\mathrm{cm} \mathrm{H}_{2} \mathrm{O}$. A $10 \mathrm{ml}$ micropipette was inserted and attached with cyanoacrylate glue to measure movement of the air bubble introduced into the system with a micro syringe. Fluid pressure was applied from the coronal area in apical direction. The volume of fluid transport was measured by observing the movement of air bubbles within a 10 $\mathrm{ml}$ micropipette at 24 hours.

Previously, the system was sealed and checked for $10 \mathrm{~min}$ to ensure there was no leakage. The root was covered with water and then covered with a plastic dish to prevent evaporation. Each specimen was attached to the device, and then the system was bled by opening the joint between the water reservoir and the specimen. The air bubble was then aligned with the zero point of the $10 \mathrm{ml}$ scale. Permeability was measured as the distance that the bubble had moved by the end of 24 hours. This measurement showed the amount of fluid which had permeated through the root filling in $\mu$ l. A test was done with two additional roots, covering the apex with two layers of nail varnish, to ensure that there was no leakage or fluid movement anywhere within the device.

\section{Micro Push-out test}

Ten roots for each group were cut into four slices $1 \mathrm{~mm}$ thick, from cervical to apical direction (Cervical1 [C1], Cervical2 [C2], Middle1 [M1], Middle2 [M2]), using a $300 \mu \mathrm{m}$ thick sintered diamond wafering blade perpendicular to the long axis of the root canal at low speed with constant water cooling. The thickness of each root slice was assessed by means of a digital caliper. Each slice was subjected to compressive loading via a universal testing machine (Instron 3345, Instron Ltd, High Wycombe, UK) equipped with a $0.5 \mathrm{~mm}$-diameter cylindrical plunger. The plunger was positioned so that it only touched the root filling on loading, without touching the canal wall or introducing shear stress along the interfaces. Thus, the side with the smaller diameter of the root canal faced upwards and was aligned with the shaft that would exert pressure load on the filling (apical-coronally) until the filling was displaced. The machine was calibrated at a constant speed of $0.5 \mathrm{~mm} / \mathrm{min}$, and the maximum failure load was recorded in Newton $(\mathrm{N})$. Failure was shown by the extrusion of the intact cone of root filling from the root slice and affirmed by the appearance of a sharp drop along the load/ time curve recorded by the testing machine. The computer and software attached to the universal testing machine calculated the $\mu$ Push-out bond strength value for each specimen, and converted them into MPa by dividing the applied load by the bonded area.

The slices were examined under a stereomicroscope at 40x magnification to determine modes of failure: adhesive at filling material with dentin interface, cohesive within filling material, or mixed failure.

\section{Statistical analysis}

The normality of the data distribution in all the study groups was verified by applying the ShapiroWilk test, and Levene's test was used to explore the homogeneity of variance among groups.

Fluid movement values at 24 hours were compared among the three different sealers and filling materials by means of one-way ANOVA and Tukey's tests.

To analyze $\mu$ Push-out bond strength values, an ANOVA for repeated measures was first applied to explore the influence of the root level (within-subject factor) and sealers/filling material (between-subjects factor) on the $\mu$ Push-out bond strength to root walls. As the sample groups did not fulfill homoscedasticity requirements, nonparametrical tests were used to compare $\mu$ Push-out bond strength values among materials (KruskalWallis' and Mann-Whitney's U test) and root levels (Friedman's tests for multiple comparisons and Wilcoxon's rank tests, for pairwise comparison).

The $\mathrm{Chi}^{2}$ test was used to analyze the mode of failure distribution among groups. Differences were considered significant for $\mathrm{p}<0.05$.

\section{RESULTS}

\section{Permeability test}

Mean fluid filtration values (SD) are recorded in Table 1. The AH Plus/gutta-percha group showed the highest value and the RealSeal group the lowest value, though there were no significant differences among the three sealer/core materials. This study makes manifest that all the materials used allowed fluid to flow along the root canal twenty four hours after filling.

\section{Micro Push-out bond strength}

Descriptive statistics of the $\mu$ Push-out bond strength are given in Table 2 . 
ANOVA for repeated measures demonstrated that root level $(F=27.148, p<0.001)$, and the interaction between the root level and sealer/core material $(F=8.66, p=0.001)$, significantly affect the $\mu$ Push-out bond strength to the walls of the root canal. The sealer/core material in itself does not significantly influence the $\mu$ Push-out bond strength values $(F=2.492 ; p=0.102)$.

For the whole sample, statistically significant differences were detected with respect to the root level (Friedman's test, $\mathrm{Chi}^{2}=23.93$; $\mathrm{p}<0.001$ ). Pairwise comparisons gave differences among all levels, with increased $\mu$ Push-out values from cervical to apical except between M1 and M2 levels.

When each material was considered separately, AH Plus/gutta-percha also demonstrated significant

Table 1- Mean values and standard deviation of permeability $(\mu \mathrm{L} / 24 \mathrm{~h})$

\begin{tabular}{cccc}
\hline Groups & Sealer & $\mathbf{n}$ & Mean permeability (sd) \\
\hline I & AH Plus & 10 & $10.34(6.45)$ \\
II & EndoREZ & 10 & $8.49(5.92)$ \\
III & RealSeal & 10 & $6.20(3.82)$ \\
\hline
\end{tabular}

differences according to root level, obtaining the lowest values in slice $\mathrm{C} 1$. For RealSeal system, the $\mu$ Push-out bond strength increases apically, with $C 1 \leq C 2 \leq M 1=M 2$. EndoREZ system showed a similar behavior at all root levels $\left(\mathrm{Chi}^{2}=3.54 ; \mathrm{p}=0.315\right)$.

$\mu$ Push-out values of the three sealing systems were also compared in each level. Levels $\mathrm{C} 1$ and C2 showed no differences in terms of sealer/core materials, but there were differences between sealers at deeper levels: M1 and M2. RealSeal demonstrated greater bond strength than EndoREZ at $M 1$ level $(U=21.00 ; p=0.028)$. At $M 2$ level, EndoREZ showed a significantly lesser bond strength than $\mathrm{AH}$ Plus $(U=22.00 ; p=0.034)$ and RealSeal ( $U=13.00 ; p=0.005)$ (Figure 1$)$.

\section{Mode of failure}

The predominating type of failure for all three sealer/core materials tested was adhesive failure, followed by mixed type failures. For the whole sample, this distribution was similar among the three sealers used $\left(\mathrm{Chi}^{2}=2.45, \mathrm{p}=0.65\right)$, and the four root levels $\left(\mathrm{Chi}^{2}=4.66, \mathrm{p}=0.58\right)$ (Table 3 ).

Table 2- Means and standard (MPa) deviations of $\mu$ Push-out bond strength test. Different letters indicate significant differences in $\mu$ Push-out strength values among root levels, for each material and for the whole sample

\begin{tabular}{cccccc}
\hline Groups & Cervical1 & Cervical2 & Medium1 & Medium2 & Mean(sd) \\
\hline AH Plus & $1.69(1.18)^{\mathrm{a}}$ & $2.05(1.00)^{\mathrm{a}, \mathrm{b}}$ & $2.48(1.32)^{\mathrm{a}, \mathrm{b}}$ & $3.30(2.22)^{\mathrm{b} 1}$ & $2.38(1.14)$ \\
EndoREZ & $0.95(1.08)$ & $2.06(1.77)$ & $1.59(0.98)$ & $1.44(1.11)$ & $1.51(0.94)$ \\
RealSeal & $0.77(0.46)^{\mathrm{a}}$ & $0.94(0.68)^{\mathrm{a}, \mathrm{b}}$ & $3.41(1.88)^{\mathrm{b}, \mathrm{c}}$ & $4.59(2.34)^{\mathrm{c}}$ & $2.43(1.01)$ \\
Mean (sd) & $1.14(1.01)^{\mathrm{a}}$ & $1.69(1.31)^{\mathrm{b}}$ & $2.49(1.58)^{\mathrm{c}}$ & $3.11(2.31)^{\mathrm{c}}$ & \\
\hline
\end{tabular}

Different letters indicate significant differences in $\mu$ Push-out strength values among root levels, for each material and for the whole sample

Table 3- Failure mode. $\mathrm{n}(\%)$. Different letters indicate significant differences in $\mu$ Push-out strength values among root levels, for each material and for the whole sample

\begin{tabular}{ccccccc}
\hline Groups & Failure & Cervical1 & Cervical2 & Medium1 & Medium2 & Total \\
\hline AH Plus & Adhesive & $4(13.30 \%)$ & $5(16.5 \%)$ & $5(16.7 \%)$ & $7(23.3 \%)$ & $21(52.5 \%)$ \\
& Mixed & $5(16.7 \%)$ & $3(10.0 \%)$ & $3(10.0 \%)$ & $3(10.0 \%)$ & $14(35 \%)$ \\
& Cohesive & $1(3.3 \%)$ & $2(6.7 \%)$ & $2(6.7 \%)$ & $0(0.0 \%)$ & $5(12.5 \%)$ \\
& Adhesive & $6(20.0 \%)$ & $4(13.3 \%)$ & $8(26.7 \%)$ & $9(30.0 \%)$ & $27(67.5 \%)$ \\
EndoRez & Mixed & $3(10.0 \%)$ & $3(10.0 \%)$ & $2(6.7 \%)$ & $1(3.3 \%)$ & $9(22.5 \%)$ \\
& Cohesive & $1(3.3 \%)$ & $3(10.0 \%)$ & $0(0.0 \%)$ & $0(0.0 \%)$ & $4(10.0 \%)$ \\
& Adhesive & $7(23.3 \%)$ & $7(23.3 \%)$ & $6(20.0 \%)$ & $6(20.0 \%)$ & $26(65.0 \%)$ \\
RealSeal & Mixed & $3(10.0 \%)$ & $3(10.0 \%)$ & $3(10.0 \%)$ & $2(6.7 \%)$ & $11(27.5 \%)$ \\
& Cohesive & $0(0.0 \%)$ & $0(0.0 \%)$ & $1(3.3 \%)$ & $2(6.7 \%)$ & $3(7.5 \%)$ \\
& Adhesive & $17(56.7 \%)$ & $16(53.3 \%)$ & $19(63.3 \%)$ & $22(73.3 \%)$ & $6(20.0 \%)$ \\
\end{tabular}




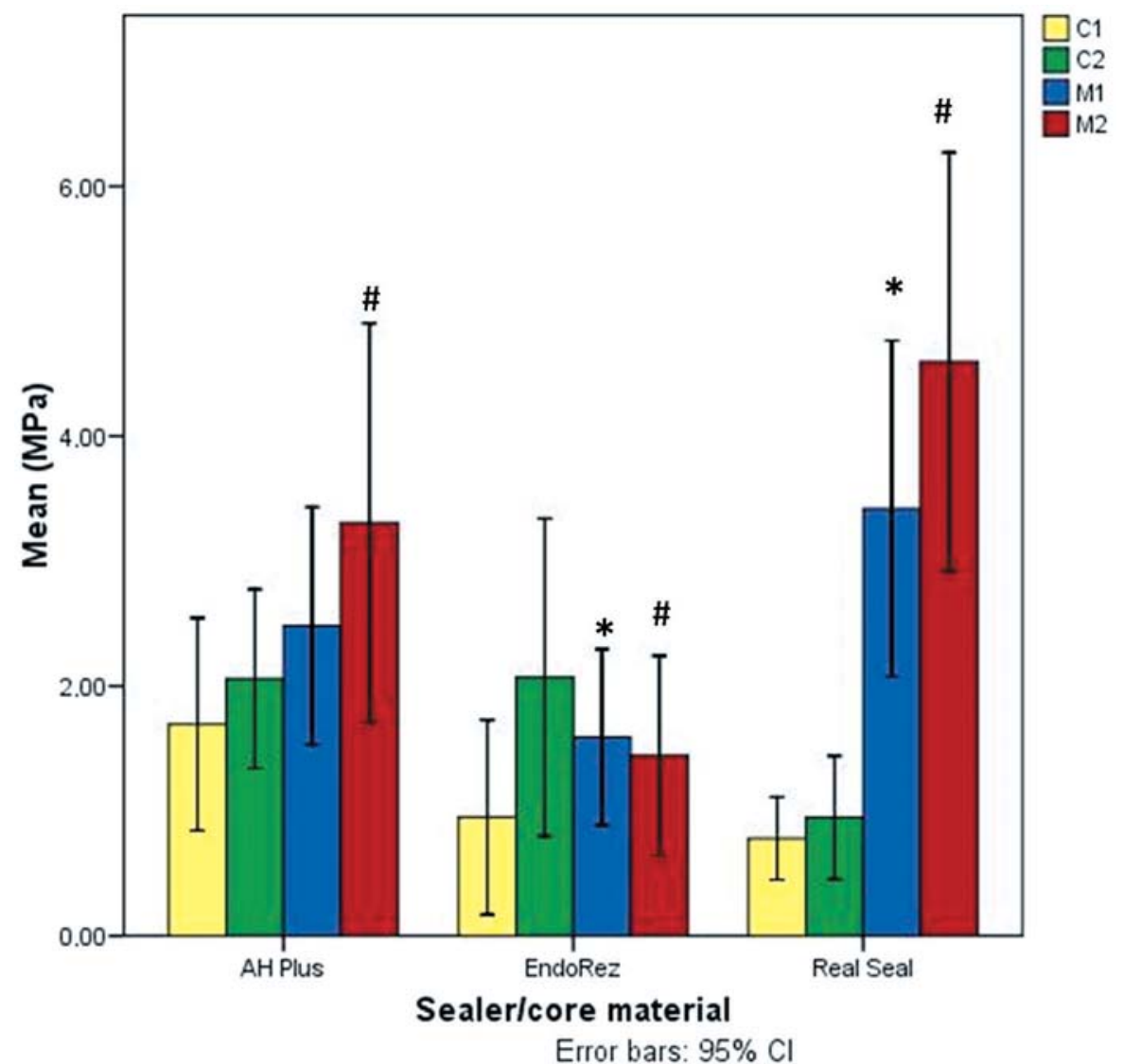

*Significant differences among EndoREZ and Real Seal at M1 level

\#Significant differences at M2 level: EndoREZ $\mu$ Push-out was significantly lesser than the rest of sealer/core materials

Figure 1- Bar chart showing $\mu$ Push-out bond strength values and comparisons among sealer/core materials in each root level (Mann-Whitney's U test)

\section{DISCUSSION}

In this study, permeability and $\mu$ Push-out bond strength were compared among roots treated endodontically with AH Plus, EndoREZ and RealSeal sealers and root canals filled using lateral condensation technique at 24 hours. The results showed that the permeability and global $\mu$ Push-out bond strength is not affected by the kind of sealer/ core material.

Microleakage studies are most commonly used to measure the ex vivo sealing ability of a root canal filling, though various methods have been proposed or recommended for evaluation of leakage ${ }^{33}$. In this study, an endodontic fluid transport model was chosen to evaluate the sealing ability of three different root canal sealers, in view of its advantages: the samples are not destroyed, it provides quantitative measurement, and the results are precise, as small volumes can be recorded $15,19,24$. We used different samples for fluid filtration and $\mu$ Push-out bond strength, to be performed at exactly 24 hours' time, thus avoiding different degrees of conversion for the sealers. We did not light-cure the coronal portion of the RealSeal sealer as recommend by manufacturer's instruction, because fast-setting light-cured resin sealers produce restriction in the flow and consequent defects or weakening of bond strength ${ }^{9}$. Moreover, the slow chemical reaction of methacrylate-based self-etching sealers may reduce shrinkage stress by means of prolonged plastic flow during the setting time of the material ${ }^{14}$. We held that such behavior would allow for complete resin infiltration into the demineralized dentin.

Our results showed that there were no statistically significant differences in the values of fluid filtration among the three sealers used in the study. It is noteworthy, however, that none of the investigated sealers/core materials achieved a tight seal at the filling-cement-root interface, a point also brought out by Vasconcelos, et al. ${ }^{32}$ (2011), allowing fluid to flow along the filled root canal twenty-four hours after filling, whereas the control showed no filtration. Currently, the clinical relevance of leakage tests in vitro has been questioned ${ }^{12}$, because it is difficult to interpret results when, in our opinion, only zero filtration can be considered a good result. Moreover, there is no correlation among various methods to evaluate microleakage, and 
the outcome of the tests depends on the evaluation method $^{12,24}$. In the absence of correlation between the ex vivo sealing ability of root filled teeth and "clinical success"20, we believe there is a threshold for microleakage values that would prove clinically relevant ${ }^{21}$.

The adhesion tests of methacrylate bond sealer to dentin have not yet been standardized. The $\mu$ Push-out test seems to be more reliable because it allows for adequate standardization of the specimens, the absence of premature failures and the variability of data distribution, while supplying a better estimation of the actual bonding effectiveness ${ }^{11}$. In all of the studies reviewed, biomechanical preparation is done with mechanical instrumentation because it is easier to perform and provides a more appropriate standardization of root canals. We used the step back technique and lateral condensation because it is still widely used throughout the world; moreover, there is no study of endodontic sealer adhesion and $\mu$ Push-out, because the small final diameter of the canal makes the $\mu$ Push-out test more difficult to perform.

In this study, we used three totally different kinds of sealers: a conventional nonbonding epoxy resinbased sealer, AH Plus; a first generation non-acidic diurethane dimethacrylate and triethyleneglycol dimethacrylate, EndoREZ; and a second generation sealer, RealSeal, based on adhesive technology with a self-etching primer and in association with a thermoplastic synthetic polyester polymer-based root canal filling material. It should be noted that the conventional nonbonding $\mathrm{AH}$ Plus/gutta-percha root filling was equal to RealSeal and EndoREZ. These discouraging results are in line with those of Ungor, et al. ${ }^{31}$ (2006), who found that the Epiphany/ Resilon combination was not superior to the $\mathrm{AH}$ Plus/gutta-percha combination. Still, most studies report that AH Plus sealer presents greater adhesion to dentin than RealSeal, regardless of root canal wall treatment ${ }^{3,7,18}$. Clinicians need to be aware that methacrylate resin-based sealers did not meet expectations regarding adhesion to root dentin, and, at this point in their development, there are no clear benefits in their use ${ }^{26}$.

The $\mu$ Push-out bond strength results were significantly influenced by dentin location. AH Plus and RealSeal obtained higher values in the deeper slices, while EndoREZ showed the lowest values, regardless of location. All sealers exhibited their low $\mu$ Push-out bond strength values in two superficial slices. This can be explained by the presence of oxygen, inhibiting sealer setting and producing a layer with low polymerization.

Because we adopted a clinical approach, we were not able to ascertain whether an increase of the adhesive strength to the apex will remain in the most apical sections. Manual instrumentation until 40 ISO width produce very limited widening of the canal in the apical section, making it impossible to perform $\mu$ Push-out tests without values having a frictional component with the canal walls. Previous authors have tested apices, but no realistic enlargements were made regarding canal preparation with drills for a post ${ }^{2}$ or using a tapered diamond bur ${ }^{18}$.

The values of the $\mu$ Push-out test are very low, consistent with results of other studies using the same methodology, indicating that the bonding of two methacrylate resin-based sealers and root dentin is much weaker than with resin-dentin bonds, 25-30 $\mathrm{MPa}^{30}$. Furthermore, the adhesive failures between sealer/dentin interface clearly suggest an inadequate level of adhesion between sealer and dentin in terms of bond strength ${ }^{2,17,27}$. This may be due to the difficulties in testing materials with great plasticity, such as gutta-percha, RealSeal and EndoREZ points; in contrast, when the test was done with sealer and no core material, values were higher ${ }^{1,2}$, suggesting failure may be traced to the sealer/material interface. In a root canal there is a highly unfavorable configuration factor (ratio of bonded to unbounded resin surfaces) ${ }^{6}$ that contributes to maximizing the polymerization stress of resin-based materials along the root canal walls; this may even exceed the bond strength of dentin adhesives to dentin, resulting in gap formation along the surfaces ${ }^{4,6,8}$. Along these lines, Souza, et al. ${ }^{28}$ (2012) report that there is a correspondence between the presence of gaps and microleakage.

Despite the material's relatively low bond strength to root dentin, it may be effective in preventing microleakage ${ }^{16}$. The main problem is that this low $\mu$ Push-out bond strength is accompanied by fluid filtration. The adhesion of resin sealer to guttapercha or core seems to play an important role in microleakage prevention, since it does not have to be at the sealer-dentin interface. Microleakage can affect the bonding of sealers to dentin by plasticization (fluids are absorbed by resins) ${ }^{8}$ and hydrolysis due to water entry in the interface. In addition, collagen degradation may occur due to host-derived matrix metalloproteinases (MMPs) in dentin that are slowly released over time when self-etching adhesives are used ${ }^{23}$. RealSeal may therefore adversely affect the longevity of bonded root canal fillings by accelerating degradation of the bond through the movement of fluid between the hybrid layer and unaffected dentin ${ }^{8,23}$.

This study reveals that global $\mu$ Push-out bond strength and permeability were not affected by different sealer/core materials: this leads us to partially accept the null hypothesis, because in the two deeper slices, RealSeal and $\mathrm{AH}$ Plus achieved higher $\mu$ Push-out bond strength than EndoREZ. We cannot confirm any clear relationship between 
permeability and $\mu$ Push-out bond strength.

\section{CONCLUSIONS}

This study makes manifest that AH Plus/guttapercha, EndoREZ and RealSeal systems allowed fluid to flow along the filled root canal in the twentyfour hours after filling. No significant differences were found among global $\mu$ Push-out bond strengths of the three sealers/filling materials; but there does exist a regional influence, by which the $\mu$ Push-out strength achieved with RealSeal and $\mathrm{AH}$ Plus to intraradicular dentin was found to be greater in the medium root slices.

\section{ACKNOWLEDGEMENT}

This investigation was supported by Research Grant no. CGL2011-25906.

\section{REFERENCES}

1- Babb BR, Loushine RJ, Bryan TE, Ames JM, Causey MS, Kim J, et al. Bonding of self-adhesive (self-etching) root canal sealers to radicular dentin. J Endod. 2009;35:578-82.

2- Barbizam JV, Trope M, Tanomaru-Filho M, Teixeira EC, Teixeira FB. Bond strength of different endodontic sealers to dentin: pushout test. J Appl Oral Sci. 2011;19:644-7.

3- Braga RR, Ferracane JL. Alternatives in polymerization contraction stress management. Crit Rev Oral Biol Med. 2004; 15:176-84.

4- Braga RR, Ferracane JL, Condon JR. Polymerization contraction stress in dual-cure cements and its effect on interfacial integrity of bonded inlays. J Dent. 2002;30:333-40.

5- Carvalho AS, Camargo CH, Valera MC, Camargo SE, Mancini MN. Smear layer removal by auxiliary chemical substances in biomechanical preparation: a scanning electron microscope study. J Endod.2008; 34:1396-400.

6- Carvalho RM, Pereira JC, Yoshiyama M, Pashley DH. A review of polymerization contraction: the influence of stress development versus stress relief. Oper Dent. 1996;21:17-24.

7- De-Deus G, Di Giorgi K, Fidel S, Fidel RA, Paciornik S. Push-out bond strength of Resilon/Epiphany and Resilon/Epiphany self-etch to root dentin. J Endod. 2009;35:1048-50.

8- De Munck J, Van Landuyt K, Peumans M, Poitevin A, Lambrechts $P$, Braem $M$, et al. A critical review of the durability of adhesion to tooth tissue: methods and results. J Dent Res. 2005;84:118-32. 9- Feilzer AJ, de Gee AJ, Davidson CL. Setting stresses in composites for two different curing modes. Dent Mater. 1993;9:25.

10- Fisher MA, Berzins DW, Bahcall JK. An in vitro comparison of bond strength of various obturation materials to root canal dentin using a push-out test design. J Endod. 2007;33:856-8.

11-Goracci C, Tavares AU, Fabianelli A, Cardoso PC, Tay FR, Ferrari M. The adhesion between fiber posts and root canal walls: comparison between microtensile and push-out bond strength measurements. Eur J Oral Sci. 2004;112:353-61.

12- Karagenç B, Gençoglu N, Ersoy M, Cansever G, Külekçi G. A comparison of four different microleakage tests for assessment of leakage of root canal fillings. Oral Surg Oral Med Oral Pathol Oral Radiol Endod. 2006;102:110-3.

13- Khedmat S, Shokouhinejad N. Comparison of the efficacy of three chelating agents in smear layer removal. J Endod. 2008;34:599-602.
14- Lawson MS, Loushine B, Mai S, Weller RN, Pashley DH, Tay $\mathrm{FR}$, et al. Resistance of a 4-META-containing, methacrylate-based sealer to dislocation in root canals. J Endod. 2008;34:833-7. 15- Miletić I, Anić I, Pezelj-Ribarić S, Jukić S. Leakage of five root canal sealers. Int Endod J. 1999;32:415-8.

16- Nagas E, Altundasar E, Serper A. The effect of master point taper on bond strength and apical sealing ability of different root canal sealers. Oral Surg Oral Med Oral Pathol Oral Radiol Endod. 2009;107:e61-4.

17- Nagas E, Uyanik MO, Eymirli A, Cehreli ZC, Vallittu PK, Lassila $\mathrm{LV}$, et al. Dentin moisture conditions affect the adhesion of root canal sealers. J Endod. 2012;38:240-4.

18- Nunes VH, Silva RG, Alfredo E, Sousa-Neto MD, Silva-Sousa YT. Adhesion of Epiphany and AH Plus sealers to human root dentin treated with different solutions. Braz Dent J. 2008;19:46-50.

19- Oliver CM, Abbott PV. An in vitro study of apical and coronal microleakage of laterally condensed gutta-percha with Ketac-Endo and $\mathrm{AH}-26$. Aust Dent J. 1998;43:262-8.

20- Oliver CM, Abbott PV. Correlation between clinical success and apical dye penetration. Int Endod J. 2001;34:637-44.

21- Özok AR, Verhaagen $B$, Wesselink PR. Improving the accuracy of a fluid transport method. Int Endod J. 2013;46:348-54.

22- Pashley DH, Derkson GD, Tao L, Derkson M, Kalathoor S. The effects of a multi-step dentin bonding system on dentin permeability. Dent Mater. 1988;4:60-3.

23- Pashley DH, Tay FR, Yiu C, Hashimoto M, Breschi L, Carvalho $\mathrm{RM}$, et al. Collagen degradation by host-derived enzymes during aging. J Dent Res. 2004;83:216-21.

24- Pommel L, Jacquot B, Camps J. Lack of correlation among three methods for evaluation of apical leakage. J Endod. 2001;27:34750 .

25- Santos JN, Carrilho MR, De Goes MF, Zaia AA, Gomes BP, Souza-Filho FJ, et al. Effect of chemical irrigants on the bond strength of a self-etching adhesive to pulp chamber dentin. J Endod. 2006;32:1088-90.

26- Schwartz RS. Adhesive dentistry and endodontics. Part 2: bonding in the root canal system-the promise and the problems: a review. J Endod. 2006;32:1125-34.

27- Shokouhinejad N, Sharifian MR, Jafari M, Sabeti MA. Push-out bond strength of Resilon/Epiphany self-etch and gutta-percha/ AH26 after different irrigation protocols. Oral Surg Oral Med Oral Pathol Oral Radiol Endod. 2010;110:e88-92.

28- Souza SF, Francci C, Bombana AC, Kenshima S, Barroso LP, D'Agostino LZ, et al. Qualitative SEM/EDS analysis of microleakage and apical gap formation of adhesive root-filling materials. J Appl Oral Sci. 2012;20:329-34.

29- Tagami J, Tao L, Pashley DH. Correlation among dentin depth, permeability, and bond strength of adhesive resins. Dent Mater. 1990;6:45-50.

30- Tay FR, Pashley DH. Monoblocks in root canals: a hypothetical or a tangible goal. J Endod. 2007;33:391-8.

31- Ungor $M$, Onay EO, Orucoglu $\mathrm{H}$. Push-out bond strengths: the Epiphany-Resilon endodontic obturation system compared with different pairings of Epiphany, Resilon, AH Plus and gutta-percha. Int Endod J. 2006;39:643-7.

32- Vasconcelos BC, Bernardes RA, Duarte MA, Bramante CM, Moraes IG. Apical sealing of root canal fillings performed with five different endodontic sealers: analysis by fluid filtration. J Appl Oral Sci. 2011;19:324-8.

33- Wu MK, Wesselink PR. Endodontic leakage studies reconsidered. Part I. Methodology, application and relevance. Int Endod J. 1993;26:37-43.

34- Zicari F, Couthino E, De Munck J, Poitevin A, Scotti R, NaertI, et al. Bonding effectiveness and sealing ability of fiber-post bonding. Dent Mater. 2008;24:967-77. 